\title{
Anomalous flexor digitorum superficialis muscle belly presenting as a mass within the palm
}

\author{
Nicholas Stephens BS, Eric Marques MD FACS, Christopher Livingston MD
}

\begin{abstract}
N Stephens, E Marques, C Livingston. Anomalous flexor digitorum superficialis muscle belly presenting as a mass within the palm. Can J Plast Surg 2007;15(1):44-46.

Anomalies of the flexor digitorum superficialis muscle are extremely uncommon and usually present as a painful mass or pseudotumour within the palm. Diagnosis may be difficult because many other soft tissue tumours (lipomas, ganglions, giant cell tumours and hamartomas) may present similarly. Magnetic resonance imaging helps to define the extent and characteristics of this anomalous muscle belly and to distinguish it from a soft tissue sarcoma, whereas plain radiographs are of little value. Three types of flexor digitorum superficialis muscle anomalies have been described, and treatment consists of subtotal or total surgical debulking of the mass if symptoms persist or if the diagnosis is in question. Most patients have complete resolution and full recovery. To date, 20 cases have been reported in the literature, usually involving the right small finger. In the present paper, the case of an anomalous flexor digitorum superficialis muscle in a 17year-old male patient's left index finger is reported. Symptoms were relieved following surgical debulking and hand-based occupational therapy.
\end{abstract}

Key Words: Anomaly; Flexor digitorum superficialis; Hand; Muscle belly; Palm; Tumour

\section{Masse dans la paume : anomalie du ventre du muscle fléchisseur commun superficiel des doigts}

\begin{abstract}
Les anomalies du muscle fléchisseur commun superficiel des doigts sont extrêmement rares et elles se présentent la plupart du temps sous forme de masses douloureuses ou de pseudo-tumeurs dans la paume. Le diagnostic peut être difficile à poser du fait que de nombreuses autres tumeurs des tissus mous (lipomes, kystes synoviaux, tumeurs à cellules géantes, hamartomes) peuvent donner le même genre de manifestations. L'imagerie par résonance magnétique aide à établir l'étendue et les caractéristiques de ce type d'anomalie du ventre du muscle et à le distinguer du sarcome des tissus mous, contrairement à la radiographie ordinaire, qui se révèle peu utile en pareil cas. Il existe trois types d'anomalie du muscle fléchisseur commun superficiel des doigts, et le traitement consiste en la chirurgie de réduction tumorale, totale ou partielle, si les symptômes persistent ou si le diagnostic est incertain. La plupart des patients connaissent une disparition totale des symptômes et un rétablissement complet du fonctionnement. La documentation fait état, jusqu'à maintenant, de 20 cas, et le plus souvent c'est l'auriculaire droit qui est touché. Il sera question, dans le présent rapport, d'une anomalie du ventre du muscle fléchisseur commun superficiel des doigts chez un adolescent de 17 ans, chez qui l'index gauche était touché. La chirurgie de réduction tumorale et l'ergothérapie axée sur les mouvements de la main ont permis un soulagement des symptômes.
\end{abstract}

A nomalies of the flexor digitorum superficialis muscle are extremely uncommon and usually present as a mass or pseudotumour within the palm. First described by MacAlister (1868) in postmortem dissections, and subsequently by Graper (1917) and Mainland (1927), this anomaly can pose a diagnostic and therapeutic dilemma (1). Most patients complain of a tender mass within zone II or III of the palm that increases in size with physical activity. Magnetic resonance imaging (MRI) may be useful in defining the extent and characteristics of the mass (2). Operative exploration and simple debulking relieve symptoms (3). To date, there have been 20 reported cases and most have involved the right hand's small finger flexor digitorum superficialis tendon $(4,5)$. We report an additional case in a 17-year-old male patient in whom the anomalous flexor digitorum superficialis muscle belly arose from the left index finger. To our knowledge, this is the fourth such case reported.

\section{CASE PRESENTATION}

A 17-year-old, right hand-dominant, black, male patient presented with a six-month history of a slowly enlarging, nontender, mobile, soft mass within zone II on the radial aspect of the left midpalm. He stated that the mass interfered with his daily exercises (push-ups) and would enlarge after any physical

activity. Past medical history was noncontributory with no reported trauma (blunt or penetrating). Recent or past foreign bodies and infection was denied, and the patient was in excellent overall medical condition. Physical examination revealed an oval mass measuring $2 \mathrm{~cm} \times 3 \mathrm{~cm}$. It appeared to be firmly attached to the index flexor tendon with movement in both the proximal and distal direction upon index finger flexion and extension. The mass had a bluish-grey discolouration and was not attached to the overlying skin. It was nonpulsatile. Phalen and reverse phalen tests were negative, and there was no Tinel sign. Two-point discrimination was normal at $5 \mathrm{~mm}$ and there were no other signs of compression median nerve neuropathy (carpal tunnel syndrome). Capillary refill was brisk and no venous congestion was present. Active and passive ranges of motion, as well as grip strength, were normal.

The differential diagnosis of a mass within the palm is extensive; thus, an MRI of the left hand was ordered. Interestingly, the final MRI report was normal; however, there was thickening within the index finger bursae and no identifiable flexor digitorum superficialis tendon (Figure 1). There was no mass or enlarged lymph nodes present.

With the diagnosis still in question, the patient chose general anesthesia and incisional or excisional biopsy depending

Division of Plastic and Reconstructive Surgery, University of Texas - Houston Health Science Center, Houston, Texas, USA

Correspondence: Dr Christopher Livingston, University of Texas - Houston Medical School, Division of Plastic and Reconstructive Surgery, 6431 Fannin Street, MSB 4.150, Houston, Texas 77030, USA. Telephone 713-500-7185, fax 713-500-0716,

e-mail Christopher.k.livingston@uth.tmc.edu 


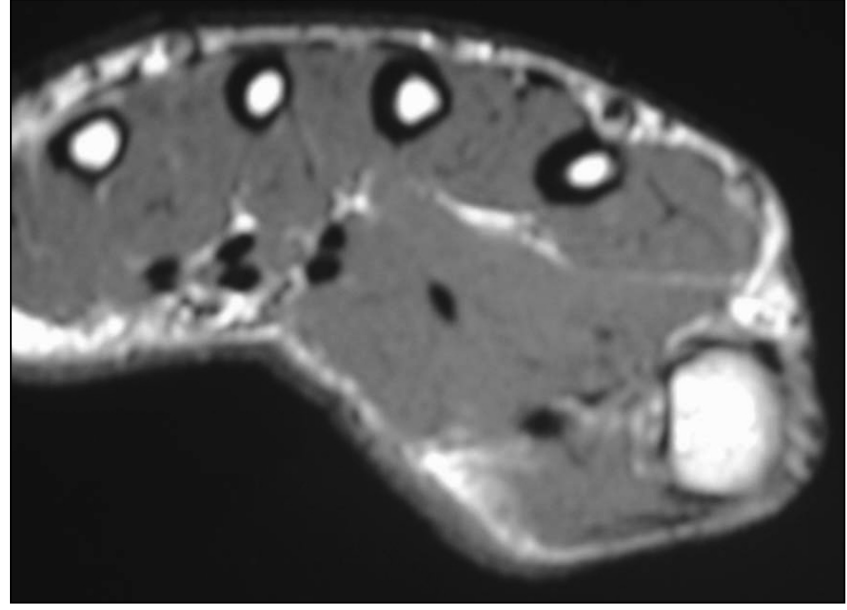

Figure 1) Magnetic resonance imaging of the left hand revealed a welldefined, non-necrotic mass within the index finger bursae without a flexor digitorum superificialis tendon. Flexor digitorum profundus and lumbrical are present and appear normal

on the intraoperative findings. Under pneumatic tourniquet control, the left palm was explored through a curvilinear incision in the thenar flexion crease extending proximally to the wrist flexion crease.

Following palmar aponeuresis division and retraction of the radial and ulnar neurovascular bundles, the distal end of the carpal tunnel was entered and a fleshy muscle belly was encountered (Figure 2). The mass was carefully dissected away from the flexor digitorum profundus tendon and its radial border lumbrical muscle, both of which appeared normal. Proximal and distal dissection revealed an anomalous flexor digitorum superficialis muscle with a musculotendinous origin within the carpal tunnel and musculotendinous insertion at the A1 and A2 pulley level. The muscle belly measured $4 \mathrm{~cm} \times$ $2 \mathrm{~cm}$ and appeared hypertrophied. There was no intramuscular tendon. Proximal traction on the muscle belly produced normal flexion of the the proximal interphalangeal joint. Innervation was provided by the median nerve through its branch to the first lumbrical and blood supply by several perforators of the superficial arch, similar to the findings by Kostakoglu et al (4). Thickened and highly vascular synovium within the carpal tunnel revealed chronic irritation synovitis; however, the median nerve was normal. All other tendons were examined and were found to be normal, and no other masses were identified. The mass was debulked by approximately $50 \%$, which allowed it to move freely within the carpal tunnel and digital flexor tendon sheath (Figure 3). Nerve and vascular supply were preserved. The wound was thoroughly irrigated with normal saline and primarily closed in two layers. A light dressing was applied and the patient was discharged home with active and passive range of motion exercises. Sutures were removed after two weeks, and range of motion returned to normal within six weeks without pain or the presence of recurrent masses.

\section{DISCUSSION}

Anomalous muscle presentations in the palm are rare and may be difficult to diagnose, primarily because of the difficulty in differentiating them from soft tissue tumours such as tendon sheath tumours, lipomas, ganglions, vascular malformations or

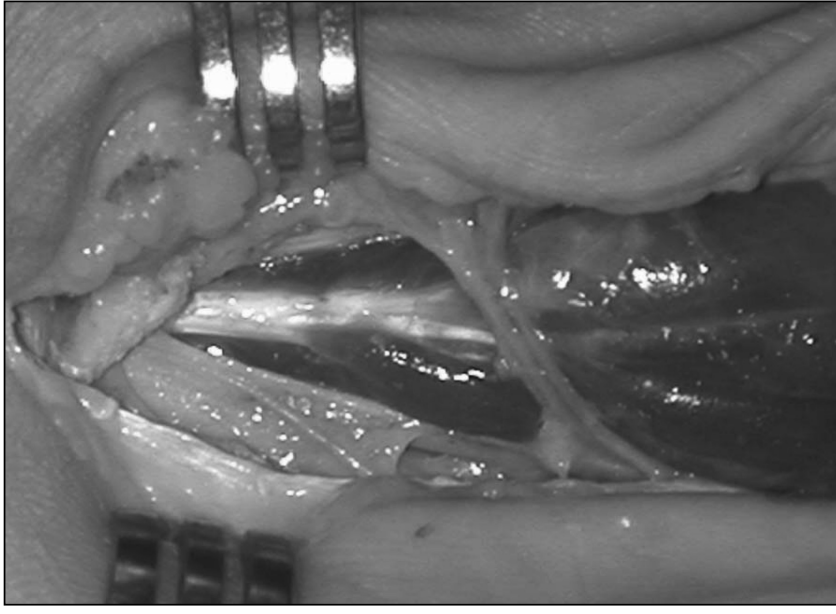

Figure 2) Anomalous flexor digitorum superficialis muscle belly within the palm (type III) dissected from surrounding neurovascular bundles, the flexor digitorum profundus tendon and lumbrical muscle

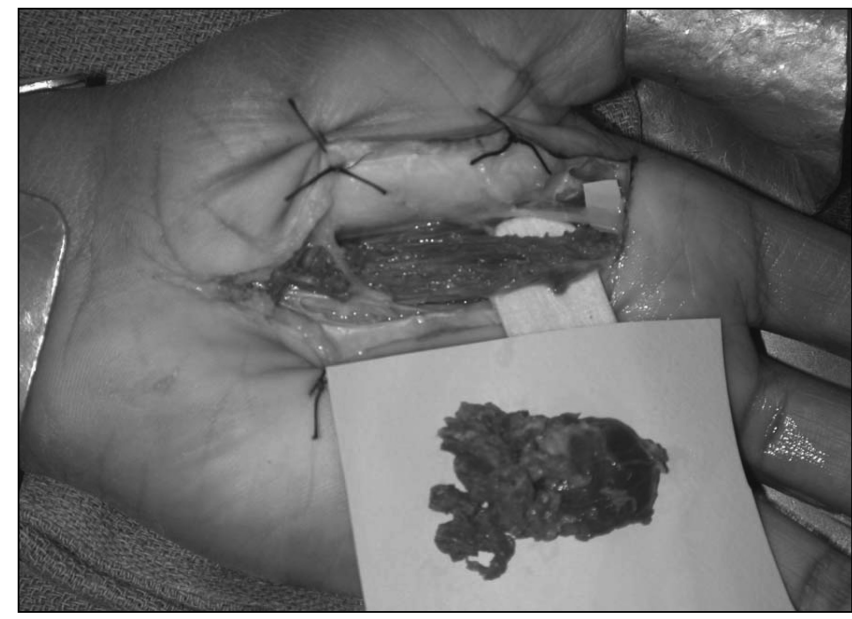

Figure 3) Subtotal surgical resection and incisional biopsy of anomalous flexor digitorum superficialis muscle belly within the palm, which allowed remaining normal muscle to move freely within the carpal tunnel, palm and fibro-osseous sheath of the index finger. The pathological specimen measured $2 \mathrm{~cm} \times 1.5 \mathrm{~cm}$

hamartomas (4), as well as foreign body granulomas or epidermal inclusion cysts. However, if the mass is soft but becomes firm with resistive flexion of the proximal interphalangeal joints, the possibility of an aberrant muscle belly should be entertained.

Anomalies of the flexor digitorum superficialis to the index finger are well documented (6-10). To date, three types of anomalies of the functional unit of the flexor digitorum superficialis that serves the index finger have been described by Probst and Hunter (11) and Christensen (12). The first type involves a muscle belly originating in the forearm and extending distally into the carpal tunnel but not further into the palm. This form can develop symptoms if the palmar belly is elongated and passes proximally under the transverse carpal ligament and into the carpal tunnel. In the second type, a digastric form exhibiting continuity by way of a tendon 
between the palmar and forearm bellies can exist. Patients with this form may present with a palmar mass or carpal tunnel syndrome (10). Finally, a third form exists in which the proximal tendon of the muscle attaches either to the base of the thenar eminence or the transverse carpal ligament. In this case, the muscle and its tendons are contained entirely in the hand and patients can present with a palmar mass that may or may not be painful. In the case of our patient, both the origin and insertion of the muscle belly were contained in the hand specifically, within the palm - similar to the description by Vichare (6) and a type III classification.

The decision to remove the muscle mass surgically should be made on an individual basis. If the muscle belly is a functional anatomical unit that serves the precise integrated flexion movement of the finger, then no surgery is required (13). Excision of the mass also may not be necessary if the patient has no symptoms of carpal tunnel syndrome (6). However, if the patient describes the mass as painful after physical activity, as did our patient, or if the aberrant muscle is in close proximity to a nerve and is causing pressure symptoms, then total or

\section{REFERENCES}

1. Elias LS, Schulter-Ellis FP. Anomalous flexor superficialis indicis: Two case reports and literature review. J Hand Surg [Am] 1985;10:296-9.

2. Sanger JR, Krasniak CL, Matloub HS, Yousif NJ, Kneeland JB. Diagnosis of an anomalous superficialis muscle in the palm by magnetic resonance imaging. J Hand Surg [Am] 1991;16:98-101.

3. Coenen L, Biltjes I. Pseudotumor of the palm due to an anomalous flexor digitorum superficialis muscle belly. J Hand Surg [Am] 1991;16:1046-51.

4. Kostakoglu N, Borman H, Kecik A. Anomalous flexor digitorum superficialis muscle belly: An unusual case of mass in the palm. Br J Plast Surg 1997;50:654-6.

5. Lillmars SA, Bush DC. Flexor tendon rupture associated with an anomalous muscle. J Hand Surg [Am] 1988;13:115-9.

6. Vichare NA. Anomalous muscle belly of the flexor digitorum superficialis: Report of a case. J Bone Joint Surg Br 1970;52:757-9.

7. Smith RJ. Anomalous muscle belly of the flexor digitorum superficialis causing carpal tunnel syndrome: Report of a case. J Bone Joint Surg Am 1971;53:1215-6. partial excision of the muscle mass is indicated $(1,7,14,15)$. Furthermore, if a neoplasm is suspected, incisional or excisional biopsy of the aberrant muscle should be performed. It is important to note that if there is no tendinous communication within the muscle mass, the risk of a delayed rupture with partial excision is a possibility and should be discussed with the patient.

\section{CONCLUSION}

Soft tissue masses within the palm are a diagnostic and management challenge. Anomalous flexor muscle bellies are extremely rare in the hand and represent arrests of development, or atavisms, in which anomalous parts retain the form they had in species lower in the evolutionary tree (16). MRI may be helpful in addition to a detailed and thorough history and physical examination. Plain radiographs are of little value. Surgical excision (partial or total) depends on the diagnosis, as well as symptoms. Complete recovery is expected, with return to normal activities and function within several weeks.

8. Hutton P, Kernohan J, Birch R. An anomalous flexor digitorum superficialis indicis muscle presenting as carpal tunnel syndrome. Hand 1981;13:85-6.

9. Das SK, Brown HG. An anomalous flexor digitorum sublinis to index finger with absent lumbrical. Br J Plast Surg 1975;28:299-300.

10. Gardner RC. Confirmed case and diagnosis of pseudocarpal-tunnel (sublimis) syndrome. N Engl J Med 1970;282:858.

11. Probst CE, Hunter JM. A digastric flexor digitorum superficialis. Bull Hosp Joint Dis 1975;36:52-7.

12. Christensen $\mathrm{S}$. Anomalous muscle belly of the flexor digitorum superficialis in two generations. Hand 1977;9:162-4.

13. Wesser DR, Calostypis F, Hoffman S. The evolutionary significance of an aberrant flexor superficialis muscle in the human palm. J Bone Joint Surg Am 1969;51:396-8.

14. Still JM, Kleinert HE. Anomalous muscles and nerve entrapment in the wrist and hand. Plast Reconstr Surg 1973;52:394-400.

15. Hayes CW Jr. Anomalous flexor sublimis muscle with incipient carpal tunnel syndrome. Plast Reconstr Surg 1974;53:479-83.

16. Elliot D, Khandwala AR, Kulkarni M. Anomalies of the flexor digitorum superficialis muscle. J Hand Surg [Br] 1999;24:570-4. 